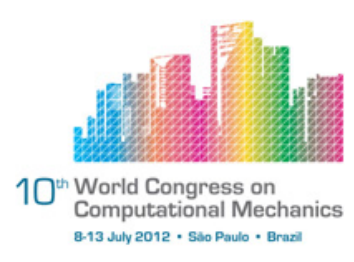

\title{
SONIC BOOM ANALYSIS UNDER ATMOSPHERIC UNCERTAINTIES BY A NON-INTRUSIVE POLYNOMIAL CHAOS METHOD
}

\author{
K. Shimoyama ${ }^{1}$, D. Ono ${ }^{1}$, A. Hashimoto ${ }^{2}$, S. Jeong ${ }^{1}$, S. Obayashi ${ }^{1}$ \\ ${ }^{1}$ Institute of Fluid Science, Tohoku University (shimoyama@edge.ifs.tohoku.ac.jp) \\ ${ }^{2}$ Japan Aerospace Exploration Agency (JAXA)
}

\begin{abstract}
This study performed the sonic boom analysis considering atmospheric uncertainties at low computational costs. Non-intrusive polynomial chaos (NIPC) method was applied to the sonic boom analysis method solving an augmented Burgers equation. NIPC can approximate statistical behavior under uncertainties from a few samples. The augmented Burgers equation can consider the rise time of sonic boom unlike the waveform parameter method, which is commonly used for sonic boom analysis. Compared to Monte Carlo (MC) method, NIPC offered equivalent accuracy for the present sonic boom analysis even with much smaller sample size. It is confirmed that this method is adequate for practical use. In addition, the present simulation results revealed that temperature uncertainty has an impact on the local rise in sonic boom pressure, and humidity uncertainty has an impact on the entire sonic boom waveform, while wind uncertainty has almost no impact. This is because temperature uncertainty affects thermal viscosity, and humidity uncertainty affects relaxation, while the present study assumed that wind direction was uniform within each atmospheric layer.
\end{abstract}

Keywords: Sonic boom analysis, Atmospheric uncertainties, Uncertainty quantification, Monte Carlo method, Polynomial chaos method.

\section{INTRODUCTION}

In the design of low-boom supersonic aircraft, the waveform parameter method [5] is commonly used to estimate the strength of sonic boom. However this method cannot consider the rise time of sonic boom, which may psychologically impact on human beings. Instead, the sonic boom analysis solving an augmented Burgers equation [1] is proposed. Unlike the waveform parameter method, this approach can consider the rise time of sonic boom because the augmented Burgers equation accounts for thermal viscosity and relaxation effects.

Furthermore, in order to evaluate sonic boom characteristics under more realistic conditions, atmospheric uncertainties should also be considered. In general, atmospheric temperature, wind velocity, wind direction, etc. are always fluctuating in a real-world situation where aircrafts are operated. Thus, if a low-boom aircraft is designed at an ideal flight condition without any uncertainties, its performance in actual flights may be lower than expected. 
Therefore, it is necessary to make the design more robust in an uncertain environment based on the analysis considering possible uncertainties, which is called uncertainty quantification $(U Q)$. A simple approach for UQ is to sample performance at a lot of conditions with different environments, and then evaluate the statistics (mean value and standard deviation) of performance. Monte Carlo (MC) method [2] is a most typical approach to do so; however, the computational time is too enormous to be allowed for practical use.

In this study, non-intrusive polynomial chaos (NIPC) method [3] was applied to the sonic boom propagation analysis solving an augmented Burgers equation for efficient evaluation of sonic boom characteristics against atmospheric uncertainties. This paper is organized as follows: Section 2 introduces the fundamentals of UQ methodologies. Then, Section 3 implements a numerical experiment in a test problem to compare the accuracy of stochastic estimations using MC and NIPC methods. Finally, Section 4 applies these methods to sonic boom analysis to evaluate the impacts of atmospheric temperature, humidity, and wind uncertainties on sonic boom signatures.

\section{UNCERTAINTY QUANTIFICATION}

UQ is the science of quantitative characterization and reduction of uncertainties in applications. Figure 1 illustrates a comparison of conventional and UQ-coupled simulation models. A conventional simulation considers the model shown in Fig. 1(a), which outputs a single value of solution $f(\boldsymbol{x})$ at fixed values of input parameters $\boldsymbol{x}$ in a deterministic way. On the other hand, a simulation coupled with UQ considers the model shown in Fig. 1(b), which assumes that input parameters $\boldsymbol{x}$ are affected by some uncertainties, represented as random variables $\boldsymbol{\xi}$, and an output solution $f(\boldsymbol{x})$ fluctuates under the input uncertainty variables $\boldsymbol{\xi}$, i.e., $f(\boldsymbol{x}(\boldsymbol{\xi}))=f(\boldsymbol{\xi})$. Using this model in a stochastic manner, UQ helps to provide a solution in a more realistic environment. The concepts of UQ have been studied and demonstrated in several problems related to computational fluid dynamics (CFD), such as shock-wave flow, turbulent flow, and reactive flow (these are well summarized in [7]).

UQ methodologies are categorized into intrusive methods and non-intrusive methods. Non-intrusive methods only require (multiple) solutions of the original (deterministic) model, i.e., users do not need to tamper with conventional simulation codes. On the other hand, intrusive methods require the formulation and solution of a stochastic version of the original model, i.e., users must be careful in modifying the simulation codes. Therefore, this paper takes up non-intrusive methods.

Moreover, the non-intrusive methods are categorized into sampling methods and polynomial chaos (PC) method. The details of these methods are described below.

\subsection{Sampling methods}

Sampling methods, shown in Fig. 2, are the most popular ways to estimate the stochastic features (mean, standard deviation, etc.) of solution straightforward by sampling and integrating output solutions given at many different points in the space of input uncertainty variables $\boldsymbol{\xi}$. MC [2] shown in Fig. 2(a) samples all points randomly, and it requires so many sample points as to ensure the accuracy and convergency of stochastic estimations. 

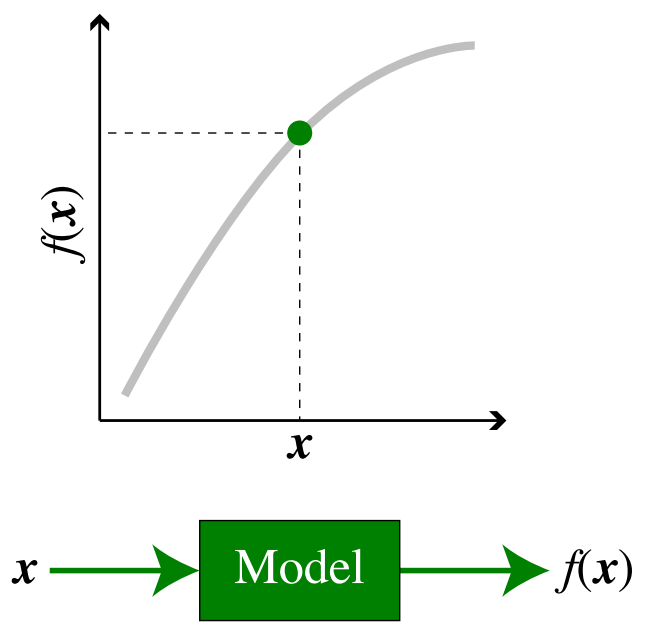

(a) Conventional model
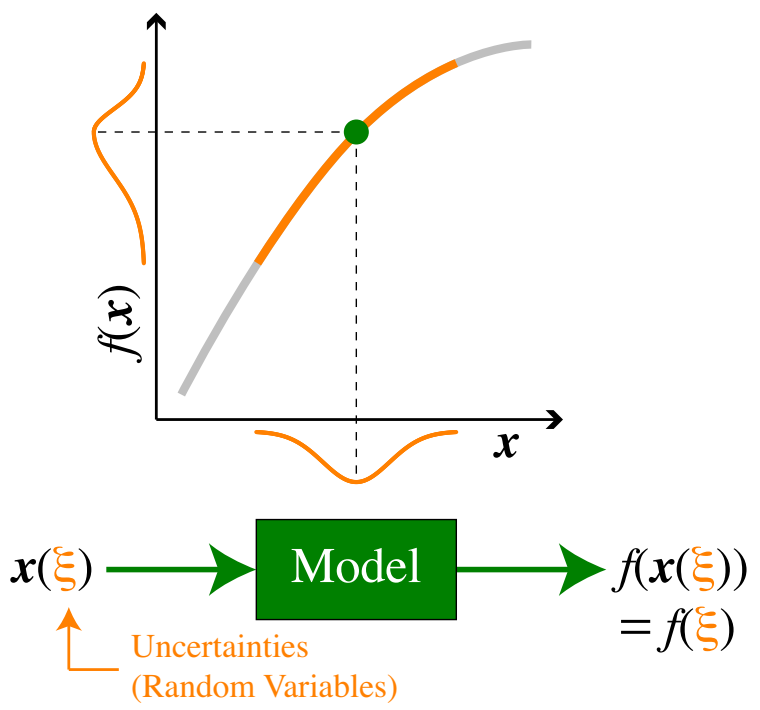

(b) UQ-coupled model

Figure 1. Comparison of simulation models.

To reduce computational cost for sampling, Latin hypercube sampling (LHS) [4] shown in Fig. 2(b) has been proposed. LHS samples a point in each equi-probability partition randomly while it does not allow overlapping partitions to be sampled for all dimensions (socalled orthogonality condition). Therefore, LHS can comprehend the whole space of $\boldsymbol{\xi}$ even with a smaller sample size than MC.

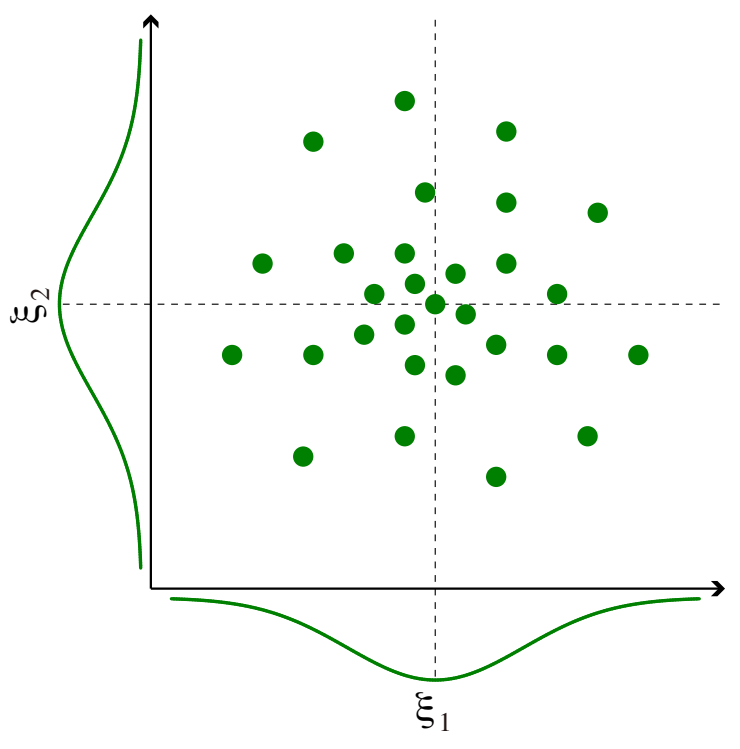

(a) Monte Carlo

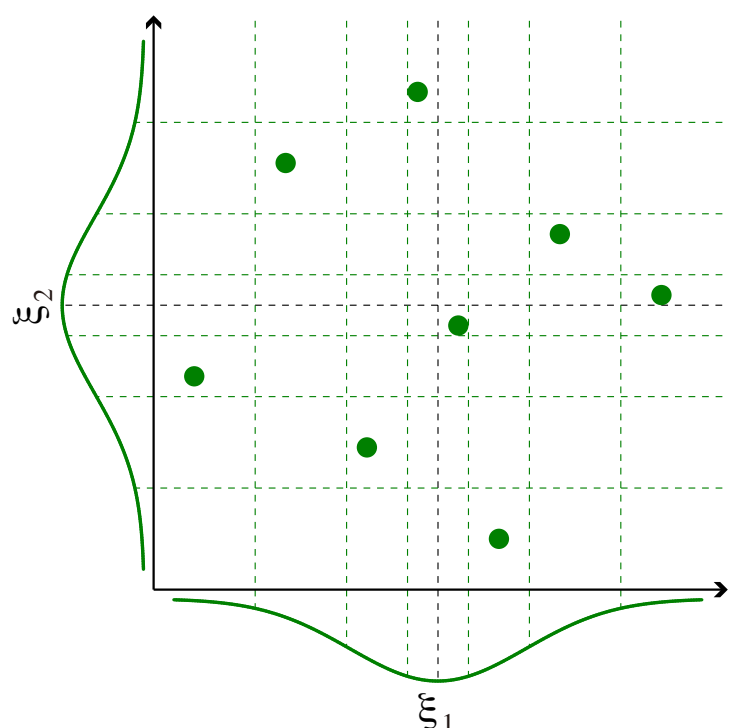

(b) Latin hypercube sampling

Figure 2. Comparison of sampling methods. 


\subsection{Polynomial chaos method}

PC method [3] approximates the behavior of an output solution $f(\boldsymbol{\xi})$ against input uncertainty variables $\boldsymbol{\xi}$ as a superposition of orthogonal polynomials:

$$
f(\boldsymbol{\xi})=\sum_{i=0}^{N} \phi_{i} \Psi_{i}(\boldsymbol{\xi})
$$

where $\phi_{i}$ and $\Psi_{i}(\boldsymbol{\xi})(i=0,1, \cdots, N)$ are the coefficient and orthogonal polynomial, which correspond to deterministic and stochastic components, respectively, for $i$-th mode. $\phi_{i}$ are obtained by sampling $f(\boldsymbol{\xi})$ at $N+1$ points with different $\boldsymbol{\xi}$ and substituting these values into the system of Eq. 1. The number of samples $N+1$ is given as

$$
N+1=\frac{(p+d) !}{p ! d !}
$$

where $p$ is the maximum order of polynomials $\Psi_{i}(\boldsymbol{\xi})$ and $d$ is the number of the dimensions of input uncertainty variables $\boldsymbol{\xi}$ (also shown in Fig. 3). A choice of polynomial $\Psi_{i}(\boldsymbol{\xi})$ is determined by a choice of the probability distribution function of input uncertainty variables $\boldsymbol{\xi}$, as listed in Table 1.

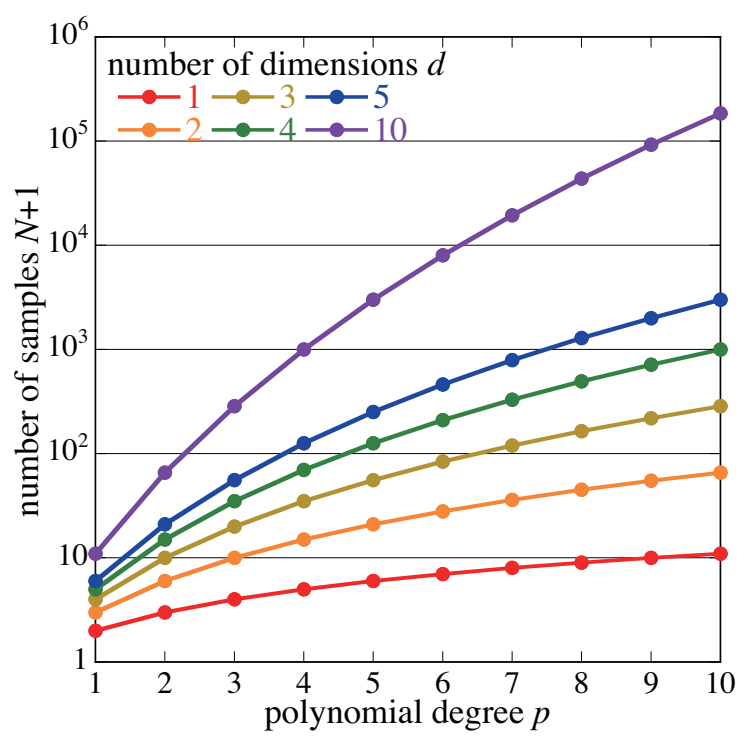

Figure 3. Number of samples required in polynomial chaos method.

For $f(\boldsymbol{\xi})$ represented as Eq. 1, the mean $\mu_{f}$ and standard deviation $\sigma_{f}$ are calculated as

$$
\begin{aligned}
E[f(\boldsymbol{\xi})] & =\mu_{f}=\phi_{0} \\
\operatorname{Var}[f(\boldsymbol{\xi})] & =\sigma_{f}^{2}=\sum_{i=1}^{N}{\phi_{i}}^{2}\left\langle\Psi_{i}^{2}\right\rangle
\end{aligned}
$$


Table 1. List of polynomials corresponding to different probability distribution functions of input uncertainty variables

\begin{tabular}{cc}
\hline Distribution $\boldsymbol{\xi}$ & Polynomial $\Psi_{i}(\boldsymbol{\xi})$ \\
\hline Uniform & Legendre \\
Normal & Hermite \\
Gamma & Laguerre \\
Beta & Jacobi \\
\hline
\end{tabular}

where the inner product of orthogonal polynomial is given as

$$
\begin{aligned}
\left\langle\Psi_{i} \Psi_{j}\right\rangle & =\left\langle\Psi_{i}^{2}\right\rangle \delta_{i j} \\
& =\int_{-\infty}^{\infty} \Psi_{i}(\boldsymbol{\xi}) \Psi_{j}(\boldsymbol{\xi}) \operatorname{PDF}(\boldsymbol{\xi}) \mathrm{d} \boldsymbol{\xi}
\end{aligned}
$$

where $\operatorname{PDF}(\boldsymbol{\xi})$ is the probability distribution function of $\boldsymbol{\xi} . \mu_{f}$ and $\sigma_{f}$ can be promptly estimated by substituting $\phi_{i}$ into Eqs. 3a and 3b without integrating $f(\boldsymbol{\xi})$ with respect to $\boldsymbol{\xi}$. Therefore, PC method is expected to estimate the stochastic features of solution even with a smaller sample size than the sampling methods.

\section{NUMERICAL EXPERIMENT}

To check the accuracy of stochastic estimations using different UQ methodologies, the present numerical experiment solves the following one-dimensional Burgers equation [6]:

$$
u_{t}+u u_{x}=\nu u_{x x}, \quad x \in[-1,1]
$$

where the initial conditions are given as

$$
u(x, 0)= \begin{cases}1 & (x \leq 0) \\ -1 & (x>0)\end{cases}
$$

and the boundary conditions are given as

$$
\begin{gathered}
u(-1, t)=1+\delta \\
u(1, t)=-1
\end{gathered}
$$

Now, $\delta$ is assumed to contain uncertainty, which is represented as a normally-distributed random variable with its mean and standard deviation of 0.05 and 0.025 , respectively. Under this uncertainty, the present experiment estimates the stochastic features of solution $\left.x\right|_{u=0}$.

Figure 6 shows the statistical values (mean and standard deviation) of solution $\left(\left.x\right|_{u=0}\right)$ against uncertainty $(\delta)$, which are estimated by MC and NIPC methods with different numbers of samples. MC statistics almost converge using 10,000 samples. NIPC statistics with more than 11 samples agree with the MC converged statistics. This result indicates that NIPC can drastically reduce the number of samples to $1 / 1,000$ while ruining the accuracy of stochastic estimations. 


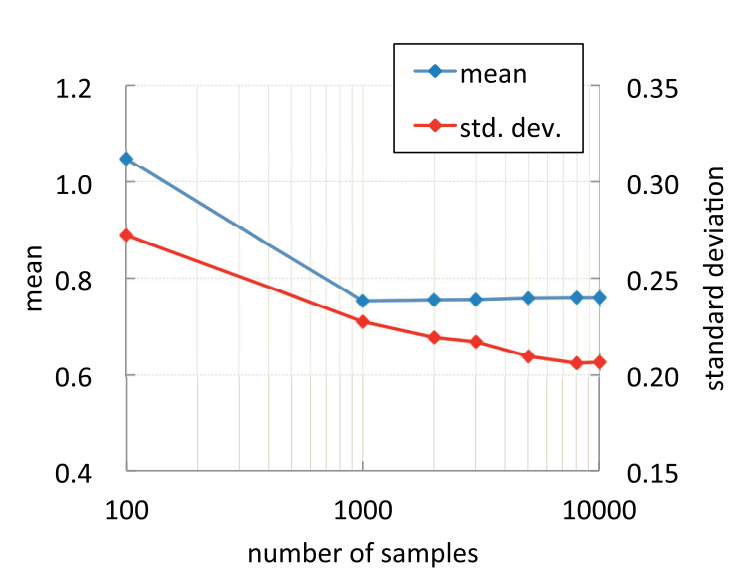

(a) Monte Carlo

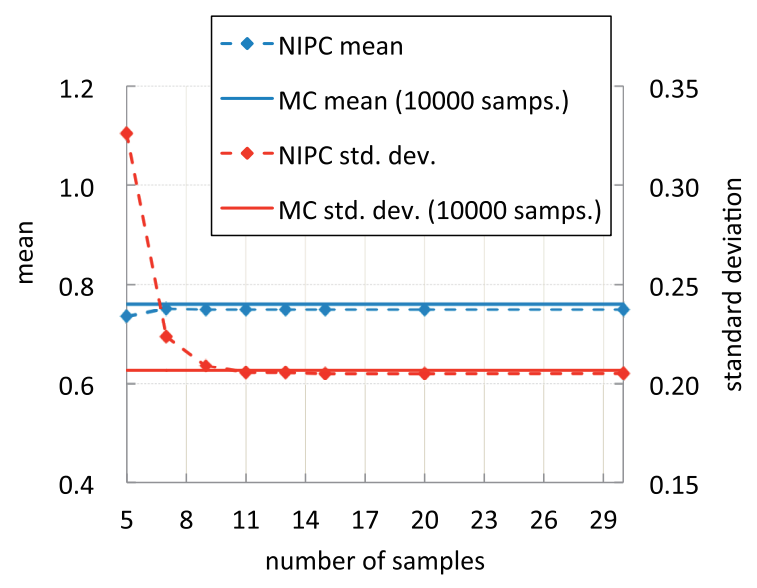

(b) Non-intrusive polynomial chaos

Figure 4. Statistical values of solution against uncertainty with different numbers of samples in numerical example.

\section{SONIC BOOM ANALYSIS}

The present sonic boom analysis solves the following augmented Burgers equation [1]:

$$
\begin{aligned}
\frac{\partial p}{\partial x}= & \frac{\beta}{2 \rho_{0} c_{0}^{3}} \frac{\partial p^{2}}{\partial t^{\prime}}-\frac{1}{2 A} \frac{\partial A}{\partial x} p+\frac{1}{2 \rho_{0} c_{0}} \frac{\partial\left(\rho_{0} c_{0}\right)}{\partial x} p+\frac{\delta}{2 c_{0}^{3}} \frac{\partial^{2} p}{\partial t^{\prime 2}} \\
& +\sum_{i} \frac{(\Delta c)_{i} \tau_{i}}{c_{0}{ }^{2}}\left(1+\tau_{i} \frac{\partial}{\partial t^{\prime}}\right)^{-1} \frac{\partial^{2} p}{\partial t^{\prime 2}}
\end{aligned}
$$

where $p$ is the sound pressure, $x$ is the ray path distance, $t^{\prime}=t-\int_{0}^{x} c_{0}{ }^{-1} \mathrm{~d} x$ is the retarded time, $A$ is the ray tube area, $c_{0}$ is the equilibrium small-signal sound velocity, $\rho_{0}$ is the ambient density, $\beta$ is the coefficient of nonlinearity, and $\delta$ is the diffusivity of sound. The first, second, third, forth, and last terms in the right-hand side of Eq. 8 consider nonlinearity effect, geometric damping effect, stratification effect (atmospheric conditions change according to altitude), thermal viscosity effect, and relaxation effect, respectively. Each relaxation process $i$ is characterized by a relaxation time $\tau_{i}$ and a small-signal sound velocity increment $(\Delta c)_{i}$.

The near-field pressure wave created by the D-SEND\#2 model (S3CM: S-cube concept model) produced in the JAXA D-SEND (drop test for simplified evaluation of nonsymmetrically distributed sonic boom) project, shown in Fig. 5, is used to analyze sonic boom propagation. The flight altitude, Mach number, and flight path angle were set to be 8000 [m], 1.3 , and $-50[\mathrm{deg}]$, respectively, and the near-field pressure wave was obtained at the location twice length of the model away from the model axis. The present analysis considers the uncertainties included in atmospheric temperature, wind (speed and direction), and humidity in August from 2000 to 2009 at the Esrange Space Center. Atmospheric temperature and wind are distributed in 17 atmospheric layers corresponding to different altitudes from 100 to 30000 [m], and atmospheric humidity is distributed in 8 layers. The mean value $\mu$ and standard deviation $\sigma$ of atmospheric properties are given in each layer separately, and the uncertain behaviors of these properties are represented as $\mu+\xi \sigma$, where $\xi$ is a normally-distributed random variable with its mean value and standard deviation of 0 and 1 , respectively. Here the present study applies a common value of $\xi$ to all layers. 


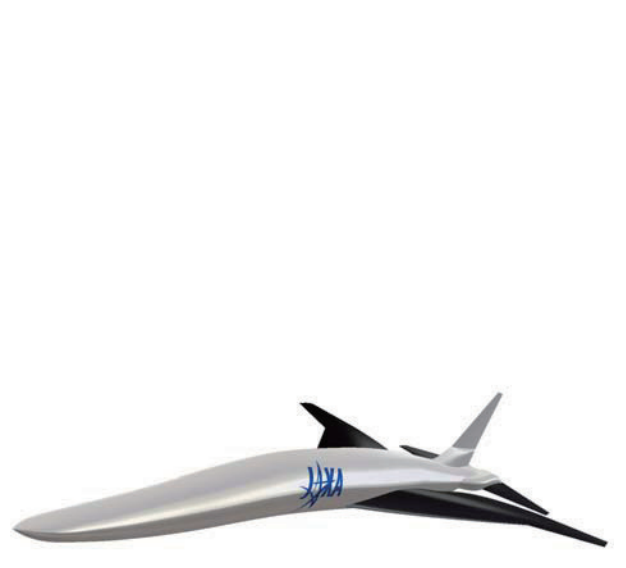

(a) Flight model

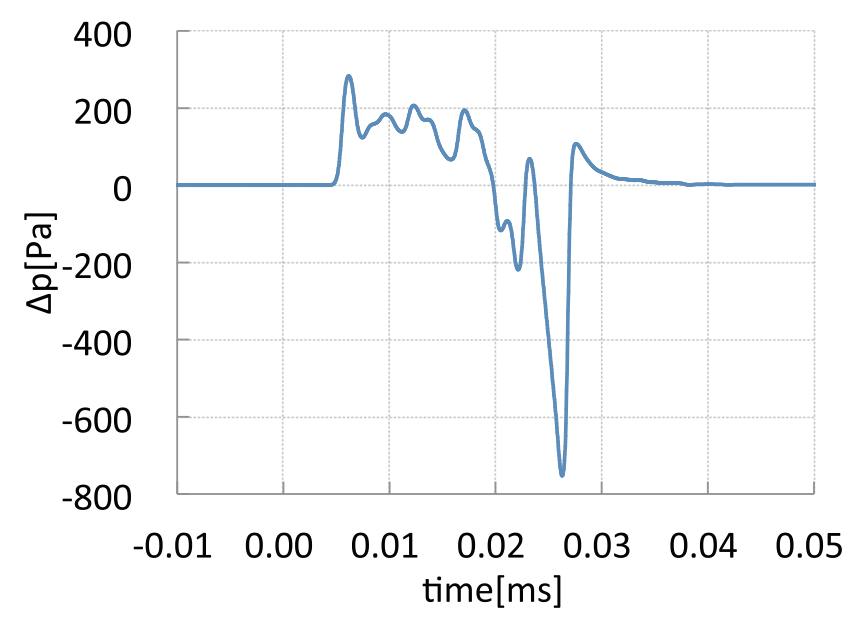

(b) Near-field pressure

Figure 5. JAXA D-SEND\#2 project.

Figure 6 shows the statistical values (mean and standard deviation) of solution (sonic boom strength at $t=0.034[\mathrm{~ms}]$ ) against uncertainty (atmospheric temperature), which are estimated by MC and NIPC methods with different numbers of samples. MC statistics almost converge using 30,000 samples. NIPC statistics with more than 11 samples agree with the MC converged statistics. This result indicates that NIPC can drastically reduce the number of samples to $1 / 3,000$ while ruining the accuracy of stochastic estimations.

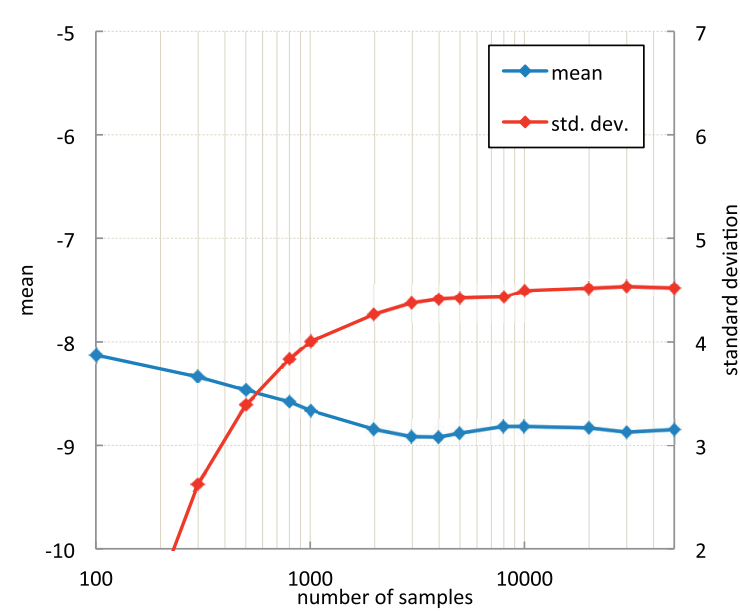

(a) Monte Carlo

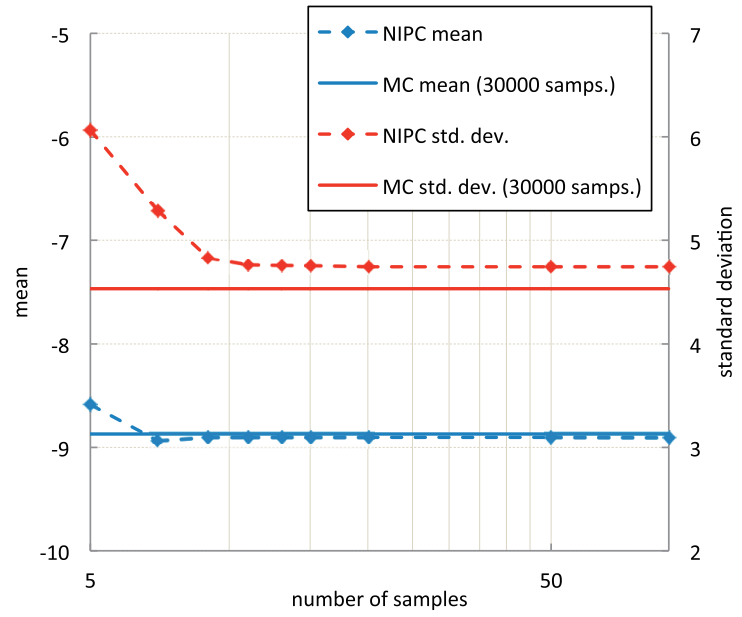

(b) Non-intrusive polynomial chaos

Figure 6. Statistical values of solution against uncertainty with different numbers of samples in sonic boom analysis.

Sonic boom waveforms considering atmospheric temperature uncertainty, humidity uncertainty, and wind uncertainty, which are obtained by NIPC with 11 samples, are shown in Fig. 7. The lines represent the mean values and the error bars represent the standard deviations of sonic boom waveforms. It is confirmed that the humidity uncertainty, shown in Fig. 7(b), has a largest impact on sonic boom. This is because atmospheric humidity significantly affects an atmospheric absorption coefficient. Next, the temperature uncertainty, shown in Fig. 7(a), has an impact on the peak pressure. This is because atmospheric temperature affects thermal 
viscosity and relaxation attenuation, which have effects on pressure gradient. Finally, the atmospheric wind uncertainty, shown in Fig. 7(c), has no effect on sonic boom. This is because the present study assumes that the wind direction is uniform within all atmospheric layers. Therefore, if the wind direction is allowed to change according to the atmospheric layers, the atmospheric wind uncertainty may have a certain effect on sonic boom.

\section{CONCLUSION}

In this study, NIPC method which can consider uncertainties at low computational cost was applied to the sonic boom analysis solving an augmented Burgers equation. It was demonstrated that, compared to MC method, NIPC could estimate the statistics of sonic boom strength with equivalent accuracy and smaller sample size. In addition, the present simulation results revealed the impacts of different atmospheric uncertainties on sonic boom signatures; temperature uncertainty has an impact on the local rise in sonic boom pressure, and humidity uncertainty has an impact on entire sonic boom waveform, while wind uncertainty has almost no impact.

\section{Acknowledgements}

The authors would like to thank the supersonic transport team in JAXA for providing the experimental data in D-SEND project.

\section{REFERENCES}

[1] Cleveland R. O., Blackstock D. T., "Waveform freezing of sonic booms revisited". NASA CP-3335, 20-40, 1996.

[2] Fishman G., Monte Carlo: Concepts, Algorithms, and Applications, Springer-Verlag, 1996.

[3] Hosder S., Walters R. W., "Non-intrusive polynomial chaos methods for uncertainty quantification in fluid dynamics". AIAA Paper 2010-129, 2010.

[4] McKay M. D., Beckman R. J., Conover W. J., "A comparison of three methods for selecting values of input variables in the analysis of output from a computer code". Technometrics 21(2), 239-245, 1979.

[5] Thomas C. L., "Extrapolation of sonic boom pressure signatures by the waveform parameter method". NASA TN D-6832, 1972.

[6] Xiu D. B., Karniadakis G. E., "Supersensitivity due to uncertain boundary conditions". International Journal for Numerical Methods in Engineering 61(12), 2114-2138, 2004.

[7] Uncertainty Quantification in Computational Fluid Dynamics, RTO-AVT-VKI Lecture, 2011. 


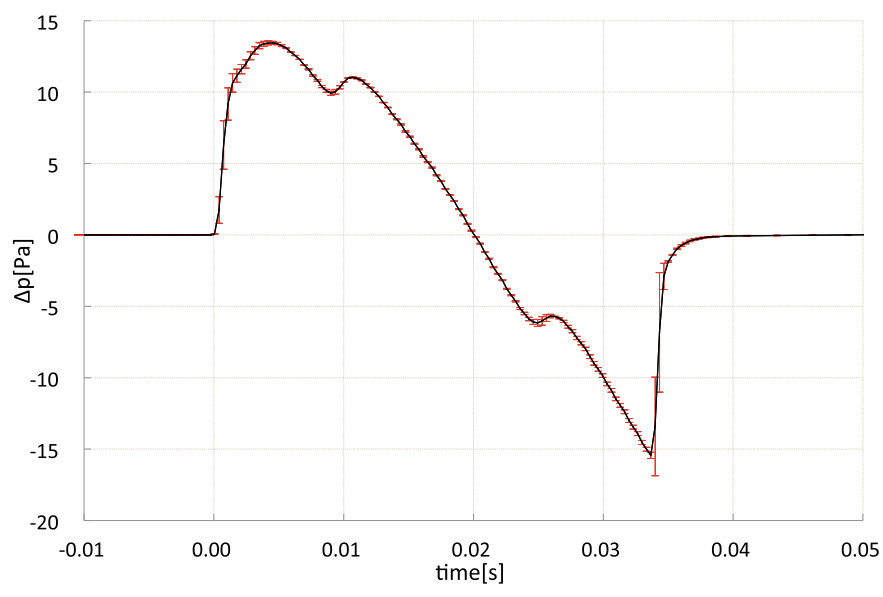

(a) Temperature uncertainty

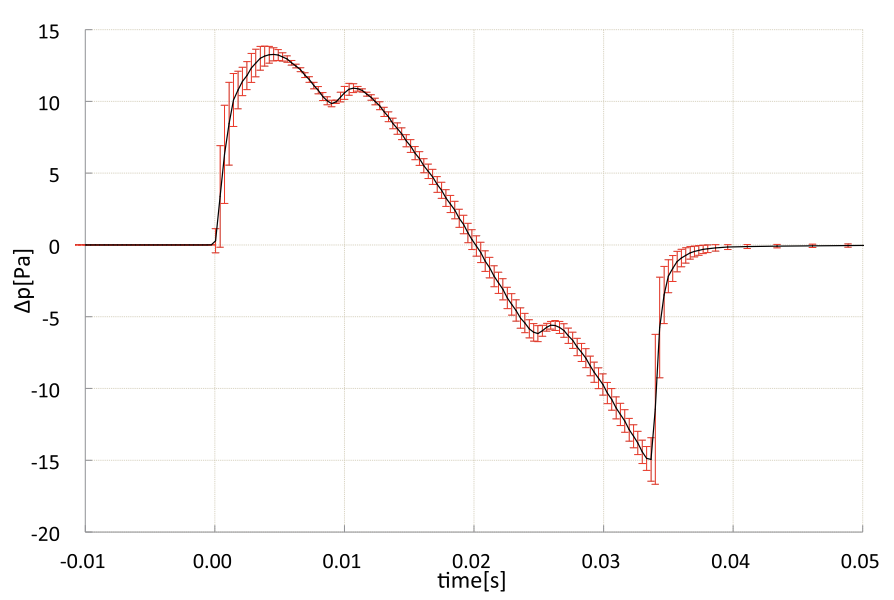

(b) Humidity uncertainty

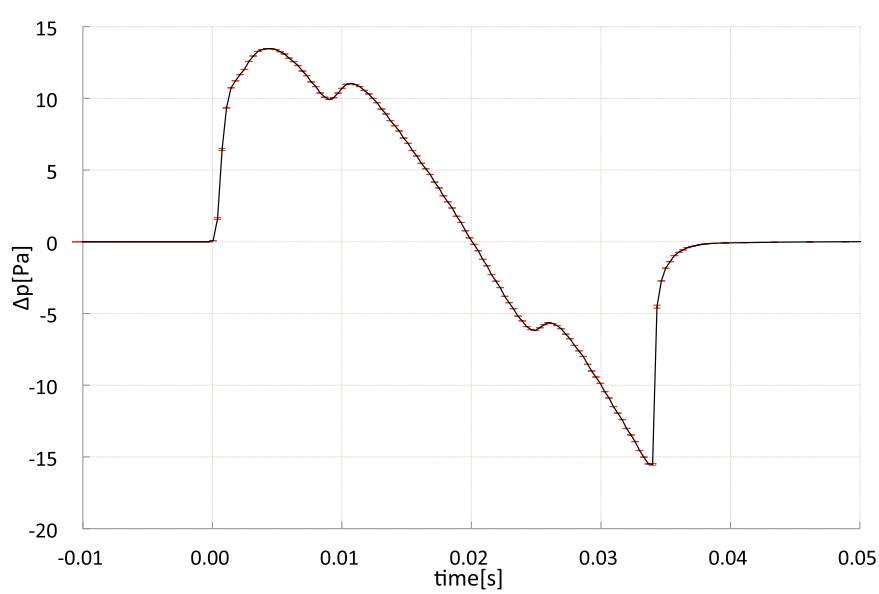

(c) Wind uncertainty

Figure 7. Comparison of sonic boom waveforms condiresing different atmospheric uncertainties. 